\title{
The best cytokine for the job
}

To the editor - Dagmar Dilloo and colleagues report intriguing results describing the generation of potent antitumor immune responses against the A20 B cell lymphoma. Our recent experience using GM-CSF to prime antitumor immune responses contradict some of their results.

Dilloo et al. report promising results following immunizing with irradiated autologous tumor cells mixed with a bystander source of interleukin-2 (IL-2) and the T-cell chemokine lymphotactin (lptn), and a considerably less promising response using granulocytemacrophage colony-stimulating factor (GM-CSF) in place of the lptn/IL-2 combination ${ }^{1}$. Histology of the vaccine site demonstrated a large infiltrating population consisting predominately of $\mathrm{CD}^{+}$ and $C D 8^{+} T$ cells and systemic depletion of these subsets resulted in the loss of anti-tumor immunity, suggesting that this strategy may work by enhancing the direct presentation of tumor antigen to the responding $T$ cells at the vaccine site. In the case of solid tumors derived from non-professional antigen presenting cells (APCs), we have demonstrated that the priming of tumor specific CD ${ }^{*}$ cytolytic T lymphocytes (CTL) occurs in response to the presentation of tumor antigens by host APCs rather than direct presentation of antigen by the tumor itself.

In contrast, B cell lymphomas are derived from cells with reasonably good APC function, and many B cell tumors have been shown to be able to process and present antigen to $T$ cells in vitro, resulting in $T$ cell activation. Such tumors express high levels of $\mathrm{MHC}$ class I and II molecules as well as having inducible expression of adhesion and co-stimulatory molecules, raising the possibility that direct $T$ cell priming may occur in response to such tumors.

To examine this issue, we explored several vaccine strategies aimed at eliciting a systemic anti-tumor immune response to the B-cell lymphoma A20 (ref. 2). Vaccination with tumor cells transfected to express GM-CSF resulted in the eradication of a pre-established systemic challenge with $\mathrm{A} 20$. In contrast, A20 cells transfected to express IL-2 or B7-1 failed to generate significant anti-tumor immunity, even though these constructs greatly enhanced the direct APC func- tion of $\mathrm{A} 20$ as measured in vitro.

We find the results presented by Dilloo and colleagues highly interesting in this regard. One question that arises is whether the effects seen in response to the paracrine production of $1 \mathrm{~L}-2+\mathrm{Iptn}$ would occur if the tumor used were derived from non-hematopoietic tissues. Do the $T$ cells that accumulate and expand in response to this combination of cytokine/chemokine still require co-stimulatory signals that may be provided by A20, but not by other tumor types? Alternatively, does some degree of tumor killing result in the liberation of tumor antigens that are picked up by infiltrating APCs present in the T-cell rich environment provided by the response to lymphotactin/lL-2?

With regard to the apparent discrepancies seen between these authors' studies of A20 GM-CSF and our own results in the same tumor model, there are several reasonable explanations. We have carefully examined the relationship between the quantity of GM-CSF produced by a vaccinating cell population and the magnitude of the systemic anti-tumor immune response generated $^{3,4}$. In several tumor systems examined, the ability to prime a successful response falls off if the vaccinating cell population produces a quantity of GMCSF less than $36 \mathrm{ng} /$ million tumor cells/24 hours. In the study presented by Dilloo and colleagues, the GM-CSF transduced fibroblasts used as a bystander cell were only making 28 ng/million cells/24 hours. Furthermore, although we and others have demonstrated that the paracrine production of GM-CSF using a bystander strategy does indeed work ${ }^{5}$, it is reproducibly somewhat less effective than when the tumor directly produces the GM-CSF. Finally, it is interesting to note that we have seen significant differences in the response to a challenge of A20 tumor given subcutaneously (as in the study by Dilloo et al.) versus an intravenous challenge. Some vaccine strategies that we have tested appear active against a subcutaneous tumor challenge with A20 but fail to eradicate a pre-established i.v. systemic tumor burden, suggesting that there may be important differences in the microenvironment of the challenge tumor that influence the results of this type of experimentation.

\section{HYAM I. LEVITSKY}

Department of Oncology

The Johns Hopkins University School of Medicine Baltimore, Maryland 21205, USA

Dilloo \& Brenner reply - We entirely agree that it is difficult to compare the potency of cytokines in murine tumor immunization studies. Relatively minor variations in the concentration or source of the cytokine, or in the route of injection of the challenge tumor may profoundly affect outcome. This problem makes it difficult to be confident that current murine models can be used to pick the "best" cytokine for a particular human tumor. Hence, our report had a different focus, namely to show that addition of a novel $T$ cell chemokine, lymphotactin, enhanced anti-tumor immune responses induced by IL-2 and GM-CSF. Since these cytokines are believed to augment immunogenicity by distinct mechanisms, our hope is that lymphotactin dependent recruitment of $\mathrm{T}$ lymphocytes to tumor sites may serve as a general means to increase cytokine mediated anti-tumor immune responses. The synergy between chemokine and cytokines is not limited to the A20 lymphoblastoid leukemia initially described, since subsequent experiments have shown identical effects in related studies using WEHI-3 (a myeloid leukemia cell line) and C1300 (a neuroblastoma-like line) (M. Grossman; unpublished data, September 1996). It remains to be seen whether these data are relevant to human disease.

Dagmar Dllloo \& Malcolm Brenner Division of Bone Marrow Transplantation and Cell and Gene Therapy Program

St. Jude Children's Research Hospital

St. Jude Place, Memphis, Tennessee 38103, USA

1. Dilloo, D. et al. Combined chemokine and cytokine gene transfer enhances antitumor immunity. Nature Med. 2, 1090-1095 (1996).

2. Levitsky, H.I. et al. Immunization with CM-CSFtransduced, but not B7-1-transduced lymphoma cells primes idiotype specific $T$ cells and generates potent systemic anti-tumor immunity. J.Immunol. $156,3858-3865$ (1996)

3. Dranoff, G. et al. Vaccination with irradiated tumor cells engineered to secrete murine CM-CSF stimulates potent, specific, and long lasting anti-tumor immunity. Proc. Natl. Acad. Sci. 90, 3539-3543 (1993).

4. Jaffe, E.M. et al. Enhanced immune priming with spatial distribution of paracrine cytokine vaccines. f. Immunotherapy 19,176-183 (1996).

5. Golumbex, P. et al Controlled release, biodegrad. able cytokine depots: A new approach in cancer vaccine design. Concer Res. 53, 5841-5844 (1993). 